\title{
Comparative Study of Machine Learning Approaches in Diabetes Prediction
}

\author{
P. Parameswari ${ }^{1}$ and N. Rajathi \\ ${ }^{1}$ Department of Computer Applications, Kumaraguru College of Technology, Coimbatore, India. \\ ${ }^{2}$ Department of Information Technology Kumaraguru College of Technology, Coimbatore, India.
}

\section{ABSTRACT}

Diabetes is a common illness that scares people around the world about their health. Biomedical research effort helps in preventing diabetics and treat it in an efficient way. There are lot of traditional systems, but it cannot handle large amount of data and it leads to problems with high levels of complexity and often it was very tedious. This research helps to design a model that can predict the risk of diabetes in patients with acceptable accuracy. Therefore, to identify diabetes in initial stage, this experiment uses machine learning algorithms, namely Random Forest, J48 as well as Multilayer Perceptron. Experiments are carried out on data collected from the UCI machine learning repository that has been gathered from patients. The impacts of all three algorithms are calculated on many scales, such as Accuracy, Precision, Recall and F-Measure. Accuracy is calculated against instances predicted correctly and incorrectly. The results obtained indicates Random forest performs well with the highest precision of 97.5 percent compared to other algorithms but J48 algorithm took minimum time to build the model.

\section{KEY WORDS: DIABETES, RANDOM FOREST, J48, MULTILAYER PERCEPTRON, MACHINE LEARNING.}

\section{INTRODUCTION}

Machine learning algorithms can learn from data and evolve from experience, without involving human beings. Learning tasks can include learning the features that map input to output, learning the hidden structure in unlabeled data, also known as example-based learning, where a class label is created from training data by comparing the new instance to memory-stored instances (Goyal et al, 2018). There are three kinds of machine learning algorithms, like Supervised Learning, Unsupervised Learning and Reinforcement Learning. To study a mapping function that converts input variables

\section{ARTICLE INFORMATION}

*Corresponding Author: parameswari.p.mca@kct.ac.in Received 12th Oct 2020 Accepted after revision 08th Dec 2020 Print ISSN: 0974-6455 Online ISSN: 2321-4007 CODEN: BBRCBA

Thomson Reuters ISI Web of Science Clarivate Analytics USA and Crossref Indexed Journal

\section{Clarivate crossef}

NAAS Journal Score 2020 (4.31) SJIF: 2020 (7.728)

A Society of Science and Nature Publication,

Bhopal India 2020. All rights reserved.

Online Contents Available at: http//www.bbrc.in/

Doi: http://dx.doi.org/10.21786/bbrc/13.11/10 into output variables, Supervised Learning utilizes labelled training data (Priyanka et al, 2020a \&t 2020b). Instance -based learning does not create an abstraction from specific instances. Two ways of classification and regression are available for Supervised Learning. Classification is used in the form of categories to predict the result of a given sample data (Parameswari et al, 2015a \& 2015. b). Unsupervised learning models are used where there are input variables and no corresponding output variables to model the basic structure of the data and are used in unlabeled training data. The reinforcement learning algorithm allows agent to decide the best next step by learning patterns that maximize a reward based on its present state (Sriram et al, 2020).

Diabetes occur when the body is not capable to produce insulin properly, which enables the body to absorb glucose as a cellular fuel and use it. This leads to a persistent increase in blood glucose levels and other abnormalities, leading in turn to the development of complications of the disease. Type I diabetes and type 2 diabetics are the common forms of diabetes. In type

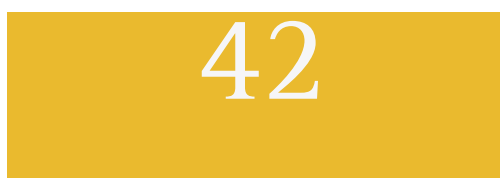


I, the immune system initiated a misguided attack and destroyed the pancreas beta cells (Rehman et al, 2020). Approximately 5\% of people with diagnosed diabetes are affected and it develops mostly during childhood. The body becomes immune to insulin and destroys the development of insulin in type II diabetes, which affects most organs. It is very important to predict diabetics at an early stage. There are many computer intelligence methods available that help with the available data sets to carry out analysis.

Related Works: The combination of fuzzy logic along with artificial neural networks and case-based reasoning for information engineering techniques has been proposed. Modified co-occurrence and cluster-based mean mode technique was implemented to manage mixed data types that can be used for any type of system (Sokolovska et al, 2018). Optimization of the updated fuzzy ant-miner designed for medical diagnostic efficacy. There are a variety of methods used to produce rules from the medical domain database (Priyanka et al, 2020c). In order to perform a diabetic prediction process, a modified and improved form of artificial bee colony algorithm have been used with an evolutionary algorithm to construct a classification system that allows doctors to make decisions. It is proposed to apply an approach that generates association rules on numeric data to medical data. Predictive Apriori diabetic prediction algorithms have been suggested by generate association rules.

In addition, there is also a greater risk of infection in people with diabetes. In most of the developed nations, diabetes is a leading cause of cardiovascular disease, blindness, kidney failure and lower levels of diabetes (Sierra-Sosa et al, 2019). Development of predictive models in predicting diabetics using risk factors is very significant. Traditional approaches have been proposed by several studies as predictors. Algorithms like Naive Bayes , Logistic Regression and Random Forest assessed the dataset and Random Forest was found to have the highest accuracy on this dataset which we used for this research. Many research come out with prediction of disease by generating rules by using Enhanced Apriori (EA) algorithm with minimum execution time and with better results comparing to the traditional algorithm.

Proposed Methodlogy: For the healthcare field, the Machine Learning Algorithm has tremendous potential as it allows health systems to use medical data for research and improve healthcare at reduced costs. Data mining techniques are useful in the prediction and diagnosis of different health issues, such as heart disease, diabetes, cancer, skin disease and so many more, when applied to health care (Parameswari \&Manikantan 2017). A more detailed way in which the information was used to predict diabetics at an early stage was discussed in this part. The proposed system forecasts the diabetics of a individual based on questions and answers provided to the prediction system. The impacts of all three algorithms are calculated on many scales, like Precision, Accuracy, F-Measure, and Recall. Accuracy is calculated against correct and incorrectly predicted instances (Priyanka EThangavel, 2020).

Random Forest Algorithm: Random Forest is a supervised learning algorithm used for both classification and regression. But it is, however, primarily used for problems with classification. As inferred, a forest is made up of trees, and more trees make the forest stronger. Similarly, the random forest algorithm produces decision trees on data samples and then gets the prediction from every one of them and picks the best solution by polling. It is an ensemble strategy that is stronger than a single decision (Parameswari et al, 2015c).

1. Begin by selecting random samples from a specific dataset.

2. For every sample, this algorithm will create a decision tree. Then, from any decision tree, it will get the prediction result.

3. For each predicted outcome, voting will be carried out at this point.

4. Eventually, as the final prediction outcome, pick the most voted prediction result.

J48 Algorithm: In order to construct a trimmed C4.5 decision tree, Quinlan's C4.5 algorithm updates J48. All aspects of the data are divided into minor subsets based on a decision. J48 looks at the structured data that really results in the information being broken by selecting an attribute. The attributes are used to obtain extremely structured knowledge. By the algorithm, the minor subsets are returned (Nagata et al, 2018). When a subset has a position with a similar class, the split strategies end.

It functions as follows.

1. If the instances belong to a similar class, the leaf is labelled with a similar class.

2. The possible data will be measured for each attribute, and the benefit in the data will be taken from the attribute test.

3. Eventually, based on the current selection criteria, the best attribute will be picked.

Multilayer Perceptron: In Artificial Neural Network (ANN), feed forward neural network class is the Multilayer Perceptron (MLP). The term MLP is applied ambiguously, for any feed-forward ANN, often specifically to mention networks made up of multiple perceptron layers. Multilayer Perceptron is often referred to colloquially as vanilla neural networks, particularly when they have a single hidden layer. The MLP consists of three main layers that is, input layer, hidden layer, and the output 
layer (Mir and Dhagae 2018). Every node is a neuron that utilizes a nonlinear activation function, except for the input nodes. MLP uses a supervised learning approach to back-propagation training. MLP is distinguished from a linear perceptron by its several layers and non-linear activation. It can discern data which cannot be separated linearly.

\section{RESULTS AND DISCUSSION}

The Weka tool is used in this research work to complete the experiment. Weka is a software tool that includes a collection of different machine learning methods for data classification, clustering, regression and visualization. One of the key advantages of using Weka is that it can be customized to suit the requirements. The main objective of this research is to predict diabetes-affected patients. We have used the algorithm J48 with modified Weka. Updated Weka offers various types of data file classification test choices, such as user training set, test set given and cross-validation. The 10-fold crossvalidation data is chosen.

Table 1. Data Set Description

\begin{tabular}{|c|c|c|}
\hline S.No & Attribute & Description \\
\hline 1. & Age & Patient Age \\
\hline 2. & Gender & Male/Female \\
\hline 3. & Polyuria & Sign of diabetics \\
\hline 4. & Polydipsia & Increase in Thirst \\
\hline 5. & Weight loss & $\begin{array}{c}\text { Patient having } \\
\text { sudden weight loss }\end{array}$ \\
\hline 6. & Weakness & $\begin{array}{c}\text { Happens if cells don't have an } \\
\text { adequate amount of glucose }\end{array}$ \\
\hline 7. & Polyphagia & Extreme hunger \\
\hline 8. & Genital thrush & Yeast infection \\
\hline 9. & Visual blurring & Temporary blurred vision \\
\hline 10. & Itching & Skin itching \\
\hline 11. & Irritability & Disturbance in patient mood. \\
\hline 12. & Delayed healing & Delay in wound healing \\
\hline 13. & Partial paresis & Weakness in muscles \\
\hline 14. & Muscle stiffness & Feeling tightness in muscles. \\
\hline 15. & Alopecia & Disorder that is the reason for \\
& & hair fall. \\
\hline 16. & Obesity & Unnecessary body fat \\
\hline
\end{tabular}

Data Set Description: Data was obtained using direct surveys from patients at Sylhet Diabetes Hospital in Sylhet, Bangladesh, donated to the UCI repository. The suggested approach that is taken from the UCI Repository is evaluated. This dataset contains medical descriptions of male and female patients in 520 instances. In the dataset, there are 16 attributes where the value of one class 0 is viewed as diabetes negative and the value of 44 another class 1 is viewed as diabetes positive. Dataset in this research work has 520 instances and sixteen attributes namely and all the 16 attributes are described in the Table 1.

Table 2. Accuracy Measures

\begin{tabular}{|l|c|c|}
\hline Algorithm & $\begin{array}{c}\text { Correct } \\
\text { Predictions }\end{array}$ & $\begin{array}{c}\text { Incorrect } \\
\text { Predictions }\end{array}$ \\
\hline Random Forest & 507 & 13 \\
\hline $\begin{array}{l}\text { Multilayer } \\
\text { perceptron }\end{array}$ & 501 & 19 \\
\hline J48 & 499 & 21 \\
\hline
\end{tabular}

Table 3. Prediction Results

\begin{tabular}{|l|c|c|}
\hline Algorithm & Accuracy & $\begin{array}{c}\text { Time (Model } \\
\text { Building) }\end{array}$ \\
\hline Random Forest & 97.5 & 0.14 \\
\hline $\begin{array}{l}\text { Multilayer } \\
\text { perceptron }\end{array}$ & 96.3 & 0.78 \\
\hline J48 & 95.9 & 0.01 \\
\hline
\end{tabular}

Figure 1: Accuracy Measures of different algorithm in manipulations

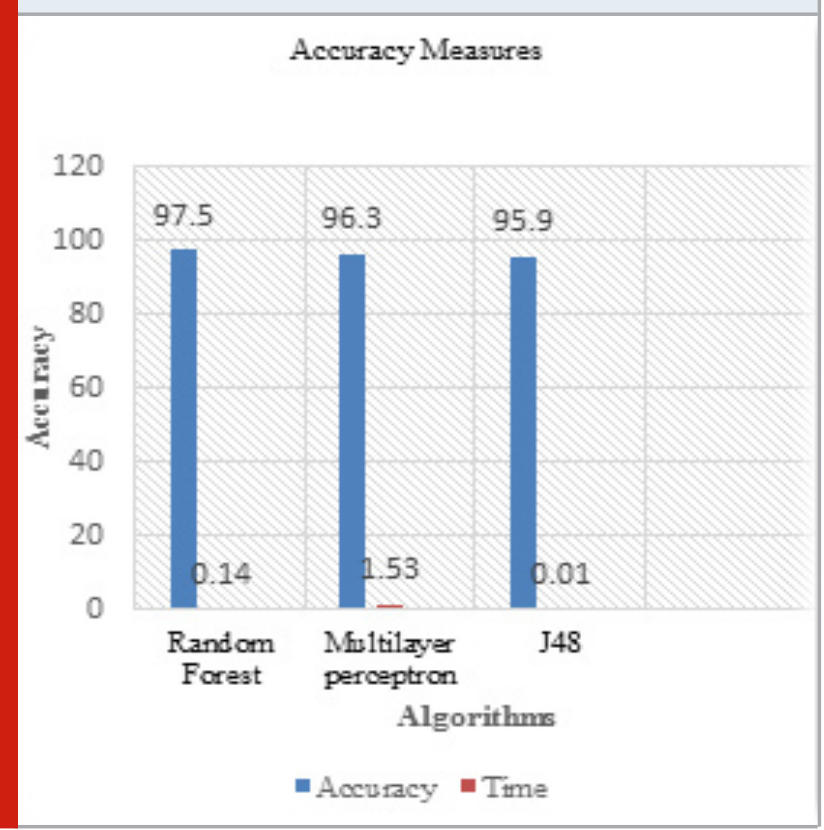

In Figures 1, 2 and 3, the pictorial representations of the prediction results and the time taken to construct the computational model of the three algorithms were given and the results are discussed in Table 2, 3, and 4. Where the difference in time between all three algorithms can be seen. The impacts of all three algorithms were evaluated on different scales such as Precision, Accuracy, 
F-Measure, and Recall. Precision is measured against exact and wrongly calculated events. The results obtained indicate that Random Forest performs well with the highest accuracy of 97.5 percent compared to other algorithms, but the J48 algorithm took limited time to create the model.

Figure 2: Prediction Results of different algorithms during manipulations

\section{Prediction Results}

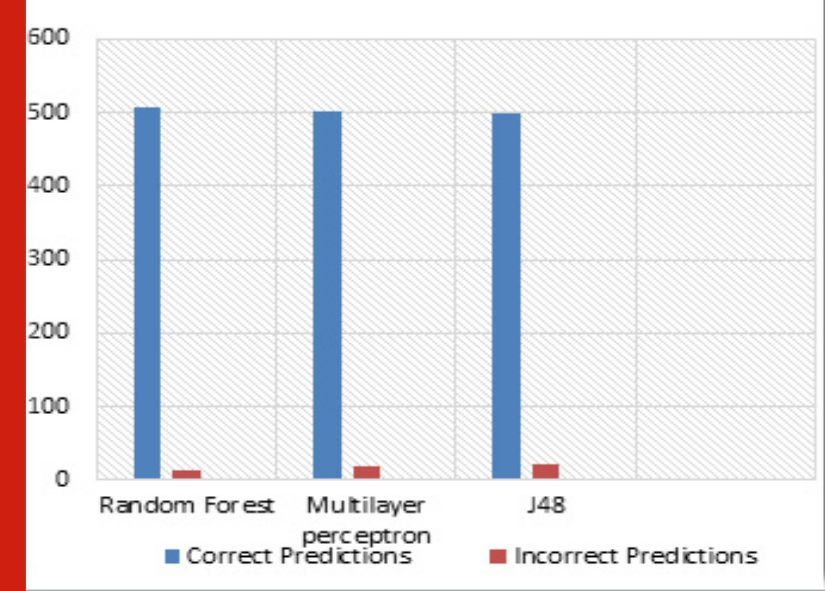

Table 4. Performance of Machine Learning Algorithms for Different Measures

\begin{tabular}{|l|c|c|c|c|c|}
\hline $\begin{array}{l}\text { Algorithm } \\
\text { Random }\end{array}$ & TPR & FPR & Precision & Recall & F-Measure \\
\hline Forest & 0.975 & 0.027 & 0.975 & 0.975 & 0.975 \\
\hline $\begin{array}{l}\text { Multilayer } \\
\text { perceptron }\end{array}$ & 0.963 & 0.038 & 0.964 & 0.963 & 0.964 \\
\hline J48 & 0.960 & 0.035 & 0.961 & 0.960 & 0.960 \\
\hline
\end{tabular}

Figure 3: Performance of Machine Learning Algorithms for Different Measures

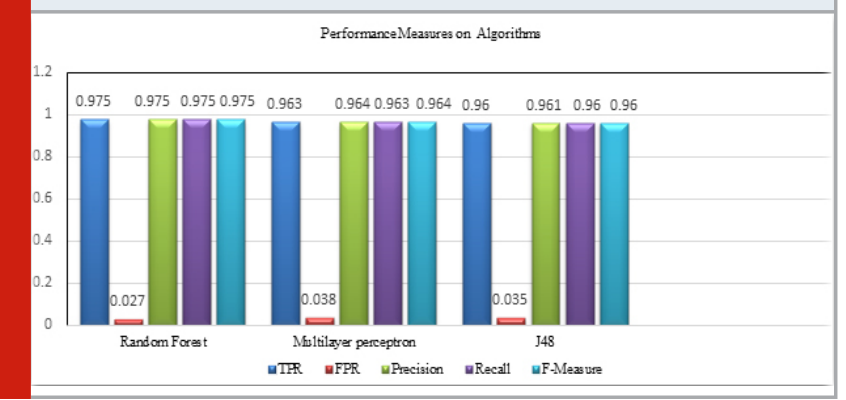

CONCLUSION

Any intelligence systems for the management of diabetics should be established with regard to the high incidence of diabetics and their impact on mortality. So the suggested system may support individuals with their symptoms to predict diabetics at an early stage. This research results in disease prediction through the use of machine learning algorithms that provide diabetic patients with knowledge. The proposed method shows that the impacts of all three algorithms are calculated by different measures such as Precision, Accuracy, F-Measure, and Recall. Compared to other algorithms, the results obtained indicate that Random forest performs well with the highest accuracy of $97.5 \%$, but the J48 algorithm took limited time to construct the model.

\section{REFERENCES}

Goyal, M., Reeves, N.D., Davison, A.K., Rajbhandari, S., Spragg, J. and Yap, M.H., 2018. Dfunet: Convolutional neural networks for diabetic foot ulcer classification. IEEE Transactions on Emerging Topics in Computational Intelligence.

Mir, A. and Dhage, S.N., 2018, August. Diabetes disease prediction using machine learning on big data of healthcare. In 2018 Fourth International Conference on Computing Communication Control and Automation (ICCUBEA) (pp. 1-6). IEEE.

Nagata, M., Takai, K., Yasuda, K., Heracleous, P. and Yoneyama, A., 2018, July. Prediction models for risk of type-2 diabetes using health claims. In Proceedings of the BioNLP 2018 workshop (pp. 172-176).

Parameswari, P. and Manikantan, D.M., 2017. GeoIntelligence System: A Frame work for agricultural improvements. International Journal of Pure and Applied Mathematics, 116(12), pp.117-125.

Parameswari, P. and Samath, J.A., 2015a. Q0S Based Web Service Ranking Using Fuzzy C-means Clusters. Research Journal of Applied Sciences, Engineering and Technology, 10(9), pp.1045-1050.

Parameswari, P., Abdul Samath, J. and Saranya, S., 2015c. Scalable clustering using rank based preprocessing technique for mixed data sets using enhanced rock algorithm. African Journal of Basic \& Applied Sciences, 7(3), pp.129-136.

Parameswari, P., Samath, J.A. and Saranya, P., 2015 b. Efficient birch clustering algorithm for categorical and numerical data using modified co-occurrence method. Int. J. Appl. Eng. Res, 10(11), pp.27661-27673.

Priyanka, E.B. and Thangavel, S., 2020. Influence of Internet of Things (IoT) In Association of Data Mining Towards the Development Smart Cities-A Review Analysis. Journal of Engineering Science \& Technology Review, 13(4).

Priyanka, E.B., Thangavel, S. and Gao, X.Z., 2020 b. Review analysis on cloud computing based smart grid technology in the oil pipeline sensor network system. Petroleum Research.

Priyanka, E.B., Thangavel, S. and Pratheep, V.G., 2020a. 
Enhanced Digital Synthesized Phase Locked Loop with High Frequency Compensation and Clock Generation. Sensing and Imaging, 21(1), pp.1-12.

Priyanka, E.B., Thangavel, S., Madhuvishal, V., Tharun, S., Raagul, K.V. and Krishnan, C.S., 2020c. Application of Integrated IoT Framework to Water Pipeline Transportation System in Smart Cities. In Intelligence in Big Data Technologies-Beyond the Hype (pp. 571579). Springer, Singapore.

Rehman, A., Athar, A., Khan, M.A., Abbas, S., Fatima, A. and Saeed, A., 2020. Modelling, simulation, and optimization of diabetes type II prediction using deep extreme learning machine. Journal of Ambient Intelligence and Smart Environments, (Preprint), pp.1-
14.

Sierra-Sosa, D., Garcia-Zapirain, B., Castillo, C., Oleagordia, I., Nuño-Solinis, R., Urtaran-Laresgoiti, M. and Elmaghraby, A., 2019. Scalable healthcare assessment for diabetic patients using deep learning on multiple GPUs. IEEE Transactions on Industrial Informatics, 15(10), pp.5682-5689.

Sokolovska, N., Chevaleyre, Y. and Zucker, J.D., 2018, March. A provable algorithm for learning interpretable scoring systems. In International Conference on Artificial Intelligence and Statistics (pp. 566-574).

Sriram, R.D. and Reddy, S.S.K., 2020. Artificial intelligence and digital tools: future of diabetes care. Clinics in Geriatric Medicine, 36(3), pp.513-525. 